\title{
PAC et transition agricole en Pologne et Roumanie : les nouveaux termes du processus
}

CAP and agricultural transition in Poland and Rumania: New terms of the process

Catherine Darrot, Béatrice Von Hirschhausen et Avec la collaboration de Marie-Luce ghib

\section{OpenEdition}

\section{Journals}

Édition électronique

URL : http://journals.openedition.org/economierurale/3257

DOI : $10.4000 /$ economierurale.3257

ISSN : 2105-2581

Éditeur

Société Française d'Économie Rurale (SFER)

Édition imprimée

Date de publication : 21 octobre 2011

Pagination : 69-84

ISSN : 0013-0559

Référence électronique

Catherine Darrot, Béatrice Von Hirschhausen et Avec la collaboration de Marie-Luce ghib, «PAC et transition agricole en Pologne et Roumanie : les nouveaux termes du processus », Économie rurale [En ligne], 325-326 | septembre-décembre 2011, mis en ligne le 17 octobre 2013, consulté le 01 mai 2019. URL : http://journals.openedition.org/economierurale/3257 ; DOI : 10.4000/economierurale.3257 


\title{
PAC et transition agricole en Pologne et Roumanie \\ Les nouveaux termes du processus
}

\author{
Catherine DARROT • Agrocampus Ouest, Rennes \\ Béatrice Von HIRSCHHAUSEN • Centre Marc Bloch, Berlin, Allemagne \\ Avec la collaboration de Marie-Luce GHIB
}

\section{Introduction}

es secteurs agricoles polonais et roumains Lprésentent au moins deux caractéristiques communes :

- ils comportent une forte masse de petites et moyennes exploitations familiales qualifiables de «paysannes »;

- à l'occasion de leur entrée dans l'Union européenne (UE), ces deux pays font l'objet d'un projet de modernisation de leurs structures d'exploitations co-piloté par leur Gouvernement et l'Europe avec le support déterminant des aides de la $\mathrm{PAC}^{1}$.

Cet article propose une confrontation du projet européen pour l'évolution des structures de production agricole en Pologne et en Roumanie avec les caractéristiques et la trajectoire spécifique des structures agricoles de ces deux pays.

La période étudiée sera celle de la préadhésion et de l'immédiate post-adhésion des deux pays. Deux corpus seront mobilisés :

- un corpus composé des textes séminaux européens et nationaux (roumains et polonais) rédigés afin d'orchestrer la mise en œuvre du second pilier de la PAC dans chaque pays : ces textes, longs et argumentés, constituent une grille de lecture du projet de développement rural élaboré en partenariat entre l'Union et les gouvernements nationaux pour chaque pays membre ;

1. Politique agricole commune.
- des données statistiques sur les structures agricoles régionales de chacun de ces deux pays, ainsi que sur la mise en œuvre des aides du premier pilier et d'un échantillon choisi d'aides du second pilier de la PAC région par région.

La comparaison de ces deux catégories de données, ainsi que la période charnière étudiée, avant et juste après l'adhésion des deux pays à l'Union, offrent une occasion unique de confronter les objectifs affichés de la PAC à la réalité de sa mise en œuvre sur le terrain : quels sont les objectifs poursuivis par l'Union pour l'agriculture de ces deux nouveaux pays membres ? Ces objectifs semblent-ils en bonne voie de réalisation, à travers un ciblage adéquat des aides vers les structures agricoles et les régions envers lesquelles l'Union affiche la plus grande volonté de soutien? Nous tenterons d'éclairer ces deux questions en interrogeant successivement les politiques menées d'abord en amont, au moment de leur formulation dans des textes programmatiques, puis en aval, au moment de leur mise en œuvre et de la distribution des aides.

Dans la première partie, nous examinerons le projet de développement agricole et rural tel qu'il s'exprime dans les textes de la PAC dans ces deux pays, en particulier pour les exploitations familiales petites et moyennes. Nous analyserons pour cela les occurrences de mots-clés et le contexte sémantique qui leur est associé. Les termes choisis, à la fois polysémiques et connotés par leurs usages dans l'histoire de la Pac 
Tableau 1. Occurrences du terme « Transition ॥ dans les textes de notre corpus

\begin{tabular}{|c|c|c|c|c|c|c|c|c|}
\hline & \multicolumn{5}{|c|}{$\begin{array}{l}\text { Différents sens attribués au terme " Transition " selon les textes } \\
\text { Et nombre d'occurrences dans chaque texte et pour chaque registre de sens }\end{array}$} \\
\hline \multirow[t]{2}{*}{$\begin{array}{l}\text { Textes } \\
\text { du } \\
\text { corpus }\end{array}$} & $\begin{array}{c}\text { Année } \\
\text { de } \\
\text { publi- } \\
\text { cation }\end{array}$ & \begin{tabular}{|c|} 
Nbre \\
pages \\
du do- \\
cument
\end{tabular} & $\begin{array}{l}\text { Nombre } \\
\text { d'occur- } \\
\text { rences } \\
\text { du } \\
\text { terme } \\
\text { Transi- } \\
\text { tion } \\
\text { pour } \\
100 \\
\text { pages }\end{array}$ & \begin{tabular}{|c} 
Sens historique \\
Période \\
faisant suite \\
à la chute du Mur \\
(1989 et après) \\
\end{tabular} & $\begin{array}{l}\text { Sens technique } \\
\text { Existence d'une } \\
\text { période de } \\
\text { transition pour } \\
\text { s'adapter à } \\
\text { I'Union } \\
\text { (contraintes } \\
\text { sanitaires, } \\
\text { administrations } \\
\text { de gestion...) }\end{array}$ & \begin{tabular}{|c|} 
Sens économique \\
et politique \\
Après l'adhésion : \\
période de \\
transition au \\
cours de laquelle \\
les aides euro- \\
péennes et les \\
exigences \\
sanitaires et \\
légales sont \\
différentes dans \\
les nouveaux \\
pays membres \\
\end{tabular} & \begin{tabular}{|c|} 
Sens \\
" européen ": \\
sens applicable \\
également à \\
l'Ouest comme à \\
l'Est \\
\end{tabular} & $\begin{array}{l}\text { Autres } \\
\text { (anecdo- } \\
\text { tique*) }\end{array}$ \\
\hline & & & & $\begin{array}{c}\text { Transition } \\
\text { vers l'économie } \\
\text { de marché } \\
\text { (but économique) } \\
\text { et la démocratie } \\
\text { (but politique) }\end{array}$ & $\begin{array}{c}\text { Transition } \\
\text { vers l'entrée } \\
\text { dans l'Union } \\
\text { européenne }\end{array}$ & $\begin{array}{c}\text { Transition vers } \\
\text { une PAC à parité } \\
\text { entre l'Europe } \\
\text { de l'Ouest } \\
\text { et les nouveaux } \\
\text { pays membres }\end{array}$ & $\begin{array}{c}\text { Transition } \\
\text { d'un système } \\
\text { technique } \\
\text { à un autre, } \\
\text { d'une enveloppe } \\
\text { budgétaire } \\
\text { à une autre, mais } \\
\text { dans le cadre } \\
\text { de la norme } \\
\text { européenne }\end{array}$ & \\
\hline $\begin{array}{c}\text { CE, } 1998 \\
\text { (Pologne) [1] }\end{array}$ & 1998 & 151 & 18 & 24 & 1 & & & 2 \\
\hline $\begin{array}{c}\text { MAFRD, } \\
2006 \\
\text { (Roumanie) }\end{array}$ & $\begin{array}{l}(2006) \\
\text { version } \\
\text { révisée }\end{array}$ & 449 & 2 & 6 & 1 & 1 & 1 & 2 \\
\hline $\begin{array}{l}\text { CE, } 2002 \\
\text { (Pologne) }\end{array}$ & 2002 & 34 & 18 & 6 & & & & \\
\hline $\begin{array}{l}\text { Adhésion } \\
\text { Pologne }\end{array}$ & 2004 & & & & & & & \\
\hline $\begin{array}{c}\text { MADR, } \\
2004 \\
\text { (Pologne) }\end{array}$ & 2004 & 166 & 5 & 2 & 5 & 2 & & \\
\hline $\begin{array}{c}\text { MADR, } \\
2005 \\
\text { (Pologne) } \\
\end{array}$ & 2005 & 206 & 7 & & 6 & 8 & 1 & \\
\hline $\begin{array}{c}\text { MADR, } \\
2007 \\
\text { (Pologne) }\end{array}$ & 2007 & 384 & 5 & & & 4 & 17 & \\
\hline $\begin{array}{l}\text { Adhésion } \\
\text { Roumanie }\end{array}$ & 2007 & & & & & & & \\
\hline $\begin{array}{c}\text { MADR } \\
2008 \\
\text { (Roumanie) }\end{array}$ & 2008 & 815 & 6 & 8 & 35 & & 1 & 6 \\
\hline
\end{tabular}

(*) Occurrence du terme dans un sens linguistique non lié au registre de cette analyse (ex : transition d'un paragraphe à l'autre...).

Source : les auteures 
depuis quatre décennies, sont révélateurs de modèles d'exploitation et de développement rural implicites que nous cherchons à expliciter dans le contexte particulier de ces deux pays d'étude.

Dans une seconde partie, nous interrogeons la mise en œuvre de ces projets dans les deux pays en examinant les volumes et les distributions d'un échantillon d'aides de la première version de la PAC. Pour ce faire nous analyserons d'abord des données statistiques portant sur les structures agricoles à l'échelle régionale. À partir d'autres macro-données portant cette fois sur les principales aides européennes sollicitées respectivement dans chacune de ces régions, nous analyserons la répartition territoriale et leur impact sur les disparités structurelles préexistantes.

\section{Quelle évolution du projet agricole européen}

Nous proposons ici de conduire une lecture organisée d'un corpus de textes rassemblé lors de la période de préadhésion puis lors de la mise en œuvre du second pilier de la PAC en Pologne et en Roumanie. Nous avons sélectionné les textes emblématiques publiés par l'Union au cours de ces deux périodes. Ceux-ci sont rédigés par les ministères de l'Agriculture de chaque pays sous le contrôle de la commission européenne et d'experts européens. Ils comportent systématiquement deux parties, découpage fécond pour la perspective que nous développons : la première propose une analyse des structures agricoles du pays, et présente une stratégie globale de développement à moyen terme. La seconde présente une batterie de mesures destinées à traduire ces enjeux en politique active de développement.

Pour en mener l'analyse, nous avons systématiquement recherché au sein de ce corpus les occurrences de termes fréquents, choisis a priori pour leur qualité descriptive, en les considérant révélateurs des attendus implicites des programmes. Pour chacun des termes retenus nous avons cherché à identifier leurs différentes acceptions à partir de leur contexte d'apparition, et étudié leur fréquence au sein des différents textes. Après avoir recherché manuellement ${ }^{2}$ les occurrences de ces termes dans les textes du corpus, nous avons composé une classification des différents sens que nous pouvions attribuer à ce terme, selon le contexte sémantique créé par chaque paragraphe où il apparaît.

\section{Transition : un principe téléologique dans le contexte d'adhésion à l'Union}

Les occurrences du terme transition ont d'abord été recherchées (tableau 1) : ce terme, amplement mobilisé pour définir l'évolution des pays d'Europe centrale et orientale depuis vingt ans, et toujours mobilisé dans la phase de préadhésion à l'Union, nous a semblé susceptible de refléter au mieux les objectifs de fond assignés à ces deux pays par l'Union.

Le nombre d'occurrences pour 100 pages du terme transition, ainsi que les divers sens qui lui sont attribués diffèrent nettement avant et après l'adhésion à l'Union. Au cours de la décennie suivant la Chute du Mur, le sens dominant de ce terme est explicitement lié à un objectif d'évolution vers l'économie de marché, assorti d'un but politique de transition vers la démocratie. Dans le texte polonais de $1998^{3}$ et dans celui de 2006 en Roumanie ${ }^{4}$, l'emploi du terme transition reste majoritairement mobilisé dans cette perspective.

2. En utilisant tout simplement le moteur de recherche proposé par le logiciel Adobe pour la lecture des documents au format pdf.

3. La Pologne est entrée dans l'Union en 2004.

4. La Roumanie est entrée dans l'Union en 2007. 
Les adhésions respectives de ces deux pays à l'Union ont manifestement marqué un double virage pour les institutions européennes et nationales co-rédactrices de ces textes : la transition vers l'économie de marché est considérée comme achevée, celle vers la démocratie est actée par l'entrée du pays concerné dans l'Union. Par conséquent les sens associés au terme transition changent, et avec eux les objectifs assignés à l'agriculture des deux pays. En 2004-2005, avec les premières versions du Sectorial Operational Program (SOP) [19] et du Plan national de développement rural (PNDR) polonais [20], un sens que nous qualifions de « technique » apparaît et domine dans les textes : il s'agit d'achever la transition vers l'entrée complète dans l'Union européenne et de rattraper les standards de l'acquis communautaire. Une période de transition est en effet maintenue au cours de la période post-adhésion, pour les mises aux normes sanitaires des laiteries et abattoirs autorisés à poursuivre leurs activités après l'adhésion ; mais aussi pour de semblables mises aux normes dans les exploitations. Dans le texte roumain de 2007 et avec le même sens, le terme renvoie à des dispositifs réglementaires et négociés mis en place par l'Union européenne pour ajuster les politiques aux spécificités du nouveau membre. De ce point de vue, la Roumanie bénéficie de l'expérience acquise au cours de la première vague d'adhésion des dix pays entrés dans l'Union en 2004.

En 2005 en Pologne, un an après l'entrée du pays dans l'Union, un sens supplémentaire que nous qualifions «d'économique et politique » apparaît dans le texte du PNDR : le terme peut aussi désigner désormais la période au cours de laquelle les aides européennes (notamment les aides du premier pilier) attribuées à la Pologne, plus faibles que dans l'Europe des 15, sont appelées à progresser chaque année jusqu'à atteindre la parité avec le reste de l'Union.

On voit enfin s'imposer un dernier sens au terme « transition » dans le texte du second
PNDR polonais de 2007 qui fait suite à la première vague d'aides pour la période 2004-2006, et qui se cale sur le calendrier de la PAC européenne pour la période 2007 2013. Il s'agit désormais de qualifier la transition technico-économique d'une exploitation d'un modèle productif à un autre sous l'impulsion de certaines mesures européennes impliquant une forme de contractualisation : transition vers l'agriculture biologique par exemple. Nous qualifions cet usage du terme de «sens européen » : il s'applique en effet indifféremment aux exploitations de l'Europe des 15 et des nouveaux pays membres.

Cette analyse montre quels objectifs ont été assignés successivement aux pays postulants à l'entrée dans l'Union. Cette perspective téléologique de convergence structurelle se décline ensuite selon les catégories d'exploitations et les régions concernées dans chacun des deux pays. L'examen de termes mobilisés pour traiter de l'avenir agricole attendu permet d'affiner l'analyse.

\section{Quelles injonctions au développement agricole et rural dans les textes?}

Nous nous intéressons ici aux occurrences de deux noyaux sémantiques, révélateurs de deux visions concurrentes du développement socio-économique agricole : celui des termes de « compétitif »/《 compétitivité » et celui des termes « durable »/« durabilité ».

Le couple de termes « compétitif/compétitivité »a été choisi parce qu'il est fréquemment mobilisé dans les textes du corpus, et révélateur d'une perspective téléologique implicite qui fait dépendre in fine la sélection des exploitations des logiques marchandes, dans une perspective où ne subsisteraient que les exploitations concurrentielles sur un marché monétisé.

Il est à placer au regard du couple des termes « durable/durabilité » dont les occurrences apparaissent conjointement dans les textes et qui renvoie, au contraire, à des discours de résistance à l'hégémonie du paradigme marchand. 
RECHERCHES

Catherine DARROT, Béatrice Von HIRSCHHAUSEN Avec la collaboration de Marie-Luce GHIB

Tableau 2. Occurrences des termes compétitifs/compétitivité et durable/durabilité dans les textes polonais et roumains

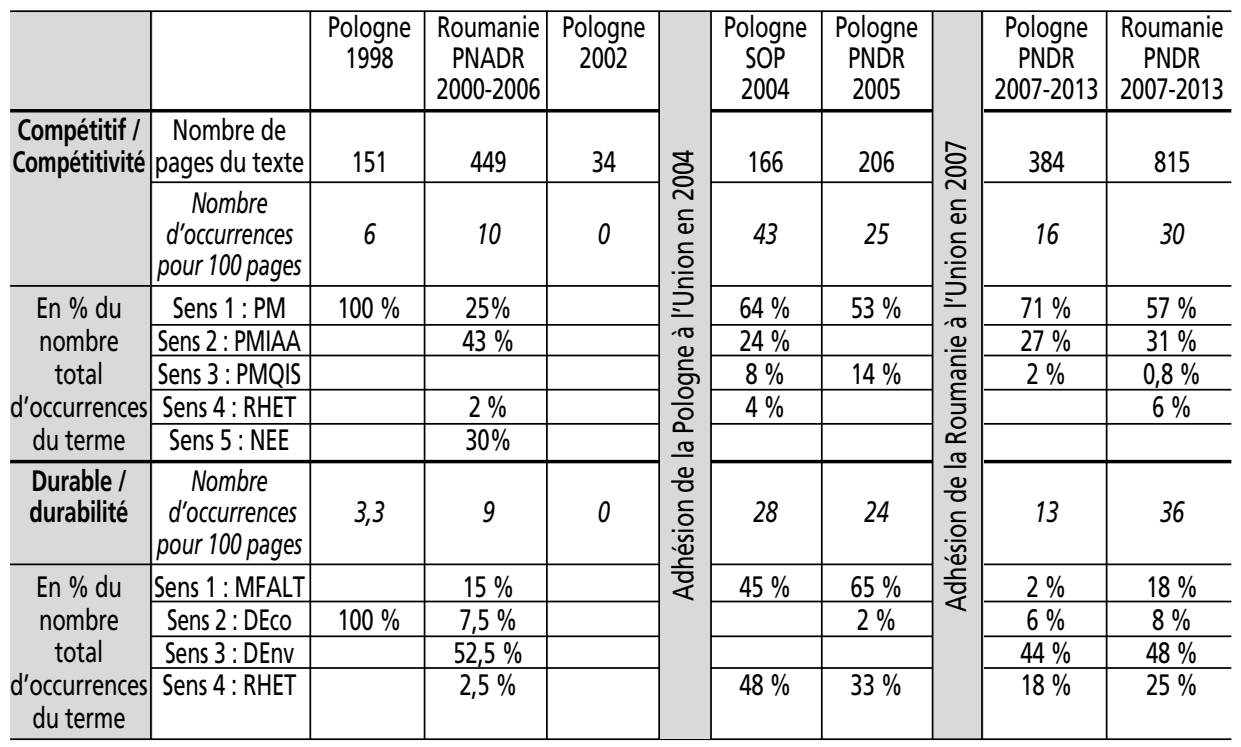

Source : les auteures

Le recours conjoint à ces lexiques concurrents reflète pour une part le grand écart des politiques européennes qui prônent l'une ou l'autre des logiques selon les types de structures agricoles en cause. Il fait aussi écho à d'autres tandems de termes tels que « moderne/modernisation », ou « structure/restructuration » que nous évoquerons aussi.

\section{Compétitif(ve)/compétitivité}

C'est avec l'intégration des deux pays dans l'Union que les termes compétitif(ve)/ compétitivité montent en puissance. Auparavant ses occurrences sont faibles.

Dans les textes polonais, le sens dominant qui leur est alors attribué est lié à la notion d'économies d'échelle en agriculture : les paragraphes dans lesquels ces termes apparaissent proposent de soutenir la conquête de parts de marché (tableau 2, sens 1, abréviation $P M$ ) par l'agriculture nationale. Cette démarche est appelée à s'appuyer sur la diminution du prix unitaire des productions (économies d'échelle par unité produite et par unité de main-d'œuvre). La compétitivité est associée à l'idée de réduction du suremploi en agriculture, et de spécialisation des exploitations agricoles dans les domaines productifs où elles peuvent se montrer les plus efficaces. Elle repose sur l'idée de qualité des productions entendue dans le sens de standardisation et de conformité avec les normes industrielles et européennes. Le projet national est ici explicitement organisé autour des principes jumeaux de restructuration et de modernisation des structures agricoles.

La seconde catégorie de sens attribuée à ce terme est liée à une perspective identique, mais appliquée cette fois non au secteur productif agricole, mais au secteur des industries agroalimentaires d'amont et d'aval (tableau 2, sens 2, abréviation PMIAA). En cumulant ces deux catégories de sens apparentées, on cerne le sens dominant attribué dans ces textes aux termes compétitif(ve)/compétitivité, ainsi que le secteur économique auquel il s'adresse : industrie et exploitations agricoles modernisées fortement liées à ce secteur industriel d'amont et d'aval. 
En Roumanie, alors que les termes compétitif(ve)/compétitivité étaient rares dans le texte du plan de préadhésion, et essentiellement mobilisés pour qualifier le nouvel environnement économique d'un marché européen ouvert (tableau 2, sens 5 , abréviation NEE) et pour traiter de la nécessité pour le secteur agroalimentaire national de se hisser à un niveau exigeant et comparable du rapport qualité/prix (sens 2), ils s'imposent dans le programme national de 2008 comme des termes clés autour du sens 1 (abréviation PM). Pour $57 \%$ des occurrences, l'apparition de ces termes dans les textes de programmation correspond à la réémergence d'un enjeu de production que la période de décollectivisation avait tendu à marginaliser. Le programme réinvestit cet enjeu à l'intérieur des logiques de marché. Il s'agit comme en Pologne d'offrir des volumes plus importants de produits capables de rivaliser en termes de prix, de qualité et de standardisation avec les produits occidentaux qui garnissent les rayons des nouveaux hypermarchés construits par dizaines par les grandes enseignes françaises ou allemandes sur le territoire à partir des années 2003-2004.

Le troisième sens attribué à ces termes (tableau 2, sens 3, abréviation PMQIS) correspond à un tout autre projet. Il s'agit toujours de garantir aux exploitations agricoles la possibilité de conquérir davantage de parts de marché. Mais il s'agira plutôt ici de fonder la performance sur une qualité des produits liée à leur identité, leur unicité, leur typicité ; ainsi que sur l'égalité sociale des chances d'accès aux infrastructures (routières, industrielles, de services...) à l'échelle territoriale ; sur l'usage multi-forme de l'ensemble des ressources (naturelles notamment) locales. Dans les textes polonais, cette version de la compétitivité s'adresse cette fois aux exploitations agricoles prises au sens de ménage (globalement) plus qu'au sens d'entreprise, et fondées sur la pluriactivité. Dans les textes roumains, les termes de compétitif/compétitivité sont associés aux termes d' " entreprises », d' " exploitations commerciales » voire même aux « exploitations de semi-subsistance » mais restent absents des chapitres traitant des exploitations de subsistance, sauf dans le cas de projets agro-touristiques pour traiter de la valorisation d'atouts territoriaux spécifiques.

Dans les textes roumains, comme polonais d'ailleurs, on observe désormais l'emploi du terme de «ferme » (ou d' « exploitation »), qui a fait son entrée dans les documents officiels avec les recensements agricoles roumains de 2000 et polonais de 2002. En s'alignant sur les catégories en vigueur dans la statistique européenne (et notamment à Eurostat), ces termes qualifient à présent l'ensemble des entreprises agricoles à l'intérieur d'un continuum allant des plus petites aux plus grandes, quel que soit leur type juridique et leurs manières très différentes d'articuler les facteurs productifs (Ghib, 2011). En outre, une évolution de l'appareil statistique est à l'œuvre pour l'analyse de l'évolution structurelle des deux pays, qui gomme littéralement la trajectoire des plus petites structures : alors que, dans les deux pays, les appareils statistiques hérités du socialisme recensaient jusqu'à l'adhésion toutes les exploitations au-delà de un ha, le suivi des structures nationales par l'Union se fait à présent, pour des raisons d'harmonisation internationale, grâce au système statistique d'Eurostat. Celui-ci ne prend en compte que les exploitations de plus de 8 UDE, gommant ainsi une part importante des petites structures pluriactives des pays de l'Est, pourtant essentielles sur le plan socio-économique. On court alors le risque d'annoncer la disparition de ces exploitations (par manque de compétitivité !) alors qu'elles n'ont disparu que des comptages statistiques (Ghib, 2011). 


\section{Durable/durabilité}

En Roumanie, les termes de durable/durabilité sont plus présents encore que les termes compétitif/compétitivité dans le texte du PNDR couvrant la période 2007. Ces termes sont en revanche présents approximativement à parité, et plus faiblement, dans le texte similaire pour la Pologne, après avoir été davantage mobilisés dans le texte du premier PNDR du pays entre 2004 et 2006.

Le sens dominant associé aux termes durable/durabilité dans l'ensemble des textes polonais (tableau 2, sens 1, abréviation $M F A L T$ ) recouvre une notion complexe liée à la multifonctionnalité des exploitations, autrement dit la présence sur une même structure de productions agricoles et d'activités rurales non agricoles, la satisfaction d'une demande sociale, des pratiques respectueuses de l'environnement et l'amélioration des conditions de vie, et de diversification des ressources économiques rurales. Ces termes sont liés explicitement à une perspective à long terme soucieuse des générations futures sur la base du triptyque social, environnemental et économique du PNUD et en référence aux stratégies communautaires de Lisbonne et de Göteborg.

Un sens presque opposé (tableau 2, sens 2, abréviation DEco) est présent dans le premier texte de préadhésion polonais de 1998, puis disparaît pour resurgir dans les textes nationaux en 2005 et 2007 . Il signe un renversement de référentiel : la durabilité de l'activité agricole n'est plus estimée qu'à l'aune de sa rentabilité économique immédiate, grâce aux parts de marchés qu'elle se montre capable de conquérir. Il exclut toute dimension environnementale et sociale, et toute notion de long terme et ramène la capacité d'une exploitation de « durer » à sa compétitivité économique immédiate. On relève également dans le PNDR roumain pour 2007-2013 dans environ $8 \%$ des cas, un usage banalisé des termes durable/durabilité, en grande partie détaché de la référence au développement durable et qui s'ins- crit dans le sens 2 repérés dans les textes polonais : la durabilité est alors simplement associée à la rentabilité économique, mais aussi à la solidité de l'investissement par différence implicite avec les projets économiques éphémères et les affaires sans lendemain qui ont caractérisé le premier capitalisme des années 1990.

Plus récemment, dans le RDR 2007 pour la Pologne, un nouveau sens de ces termes s'est imposé (tableau 2, sens 3, abréviation DEnv) : il s'agit d'une durabilité appréhendée strictement sous l'angle environnemental : préservation des ressources naturelles, de la biodiversité, sans plus aucune référence au domaine socio-économique. Le récent texte du RDR semble abandonner progressivement l'idée d'un développement rural appréhendant globalement les enjeux du développement local, pour offrir une représentation toujours plus dichotomique, des structures et des projets structurels : compétitivité économique stricto sensu, et/ou « compétitivité » environnementale. L'enjeu social n'est plus résolu qu'indirectement, à travers l'un ou l'autre de ces deux leviers. On doit également à cet objectif environnemental près de la moitié des occurrences des termes durable/durabilité dans le texte du PNDR roumain de 2007, appliqués alors à la gestion des sols, des eaux, des forêts, de la biodiversité.

On peine toutefois à séparer dans les textes les usages des termes durable/durabilité mentionnés plus haut d'usages plus rhétoriques (tableau 2, sens 4, abréviation RHET) qui émaillent le texte, associés de manière quasi mécanique aux termes de « développement », d' « agriculture », de « tourisme » sans que soit davantage précisé ce dont il s'agit.

Plus globalement en effet, tant les termes de compétitivité que de durabilité sont parfois mobilisés sans être véritablement investis d'un sens précis. Ces emplois non réfléchis témoignent du souci des auteurs de se conformer, au moins sur le plan formel, à des injonctions européennes multiples et 
souvent contradictoires en termes de développement rural. Ils peuvent aussi relever de la hâte avec laquelle certains textes, comme le premier texte du RDR polonais de 2004, furent rédigés.

Au terme de cette analyse, la question du développement agricole et rural apparaît d'abord traduite dans ces textes comme affaire de compétitivité. Elle est soumise à la capacité du secteur à trouver les moyens d'une meilleure performance. Le projet de compétitivité marchande est pensé comme autonome et prioritaire et passe par la réduction d'une masse de micro-exploitations de subsistance perçue comme «économiquement non viables », peu productives (faible productivité de la terre et du travail) et incapables de répondre à la demande potentielle de l'industrie agroalimentaire. Il se décline logiquement au sein d'un programme de concentration et de modernisation que l'on retrouve derrière l'usage de la seconde acception du terme (sens 1 PM et sens 2 $P M I A A$ ), qui désigne un projet de restructuration des exploitations transformables par intensification du capital, réduction de la main-d'œuvre agricole, concentration foncière, de rajeunissement des chefs d'exploitations et intégration sur le marché, parallèlement à la constitution, modernisation et intégration de filières. Les textes établissent une distinction entre les structures agricoles et agroalimentaires en mesure de relever le défi de la croissance productive et économique, et les autres auxquelles il faut fournir le moyen de s'inscrire dans des systèmes sociaux et territoriaux qualifiés de «durables » dans les textes.

La notion de durable/durabilité, en dépit de sa plus grande fréquence dans tous ces textes, apparaît alors dans l'ombre de l'objectif de compétitivité du secteur agroalimentaire et se trouve à la fois couplée et subordonnée au projet de compétitivité marchande, loin de la visée à long terme, englobante et holiste, de ses principes originaires. Elle est adoptée par défaut et réservée aux zones géographiques ou aux exploita- tions « non compétitive ». Ce partage entre compétitivité et durabilité participe ici d'une construction politico-économique en mosaïque, sur le plan spatial comme structurel : là où le premier projet échoue parce qu'inadapté à une vaste catégorie de petites et moyennes structures poly-productives, on fait appel au second, pour se conformer à l'ambition européenne de trouver des réponses adaptées au développement de toutes les régions ou de toutes les populations. L'usage souvent vague ou peu cohérent de ces deux termes offre la sensation d'une notion de durabilité plaquée, mobilisée et surtout rhétorique, donnant lieu à des mesures que l'on pourrait qualifier de palliatives en attendant le reflux de la catégorie d'exploitations visées.

La seconde partie de cet article nous amène à examiner comment, concrètement, les plus récents de ces principes normatifs se traduisent sur le plan opérationnel en termes d'aides publiques européennes et de répartition de celles-ci. L'analyse qui précède montre deux éléments dominants dans les textes européens : un projet de restructuration du secteur agricole vers de grandes exploitations marchandes, et un projet de soutien aux activités rurales durables pour les exploitations et les régions incapables de se soumettre au projet marchand dominant : les exploitations et les régions ciblées respectivement par ces deux catégories d'aides sont-elles effectivement celles qui s'en saisissent et en bénéficient, en particulier lorsqu'il s'agit d'aides au développement rural comportant une composante sociale ?

\section{Des aides en faveur des exploitations " compétitives "}

Sept ans après l'intégration polonaise et quatre ans après l'intégration roumaine, nous proposons d'interroger ici non plus les intentions politiques mais leurs bénéficiaires et d'observer la manière dont les fonds mis à disposition par la PAC sont 
effectivement sollicités et engagés. La question des destinataires nous permet de questionner à présent l'ajustement des dispositifs aux enjeux agricoles des deux pays.

Nous réfléchissons ici à partir des données régionales. La Pologne comme la Roumanie ont en effet hérité de leurs histoires politiques et agraires des géographies agricoles contrastées (Hirschhausen, 1997 ; Maurel et al., 2003 ; Rey et al., 2007 ; Darrot, 2008b, Ghib, 2007, 2011).

\section{Les paradoxes polonais}

Dans le cas polonais nous avons déjà suffisamment de recul pour disposer d'un ensemble assez complet d'indicateurs. Voïvodie ${ }^{5}$ par voïvodie pour l'aide unique du premier pilier et une sélection de cinq mesures emblématiques des objectifs du second pilier, nous avons relevé le nombre de dossiers d'aides demandées pour 100 exploitations. Soulignons ce point : c'est la capacité des ménages à capter ces aides que nous analysons à travers le nombre de dossiers demandés pour chaque exploitation, non le nombre de dossiers qui auraient pu être théoriquement pourvus au vu de la nature de l'aide. Nous avons préféré ce critère au montant d'aides attribué par hectare : si le second informe plus précisément sur la manière dont chaque région parvient, dans l'absolu, à capter les aides européennes, le premier semble plus intéressant pour renseigner sur la fonction sociale de ces aides : il permet d'observer quel parti les ménages agricoles parviennent à tirer de ces aides dans chaque région.

Parmi les cinq mesures choisies, trois sont issues de l'axe 1 «modernisation » du second pilier de la PAC en Pologne et reflètent le sens dominant le plus récent attaché aux termes « compétitif/compétitivité » dans les textes européens présenté dans le tableau 2 (sens 1 PM). Elles peuvent être considérées comme emblématique de la mise en

5. Voïvidie signifie « région», avec une valeur administrative. œuvre de cette perspective à travers les aides publiques :

- l'aide à la modernisation des exploitations ;

- l'aide au départ en pré-retraite ;

- l'aide aux jeunes agriculteurs.

Les deux autres aides choisies, emblématiques de l'axe 2 « soutien à la durabilité des zones rurales » du second pilier de la PAC, reflètent les deux sens dominants les plus récents des termes « durable/durabilité » mis en évidence dans notre tableau 2 :

- l'aide à la diversification des activités (sens 1 MFALT);

- les aides agri-environnementales (sens 3 DEnv).

La dimension sociale, surtout, distingue ces deux échantillons d'aides : les trois premières s'adressent à des exploitations appelées à s'agrandir et à concentrer le capital productif, les secondes s'adressent en principe à des exploitations plus employeuses, et moins tournées vers des trajectoires d'accumulation de capital matériel que vers des trajectoires technico-économiques économes et autonomes de diversification. Est-ce effectivement le cas ? Il est utile pour répondre à cette question de rechercher la corrélation entre le taux de demande de ces aides (examiné région par région) et la taille des exploitations, d'une part la quantité de main-d'œuvre par hectare et d'autre part le taux de chômage rural.

Dans les régions où la taille moyenne d'exploitation est la plus élevée, les exploitations demandent le plus souvent les aides du premier pilier, ainsi que celles au départ en préretraite et à la modernisation des exploitations. Cette dernière aide ciblée du second pilier atteint, autrement dit, manifestement sa cible sans surprise : il faut en effet être capable d'avancer le montant des investissements pour en bénéficier, l'Union ne remboursant que 18 mois plus tard. Seules les exploitations déjà grandes et nanties disposent de cette capacité de trésorerie. Cette corrélation entre taille moyenne 
Tableau 3. Indices de corrélation entre variable de structures et variable exprimant les taux de demandes d'aides

\begin{tabular}{|c|c|c|c|c|}
\hline \multicolumn{2}{|c|}{ Indices de corrélation } & $\begin{array}{l}\text { Variable de tailles } \\
\text { des exploitations }\end{array}$ & $\begin{array}{l}\text { Variable emploi } \\
\text { agricole/100 ha }\left(^{*}\right)\end{array}$ & $\begin{array}{c}\text { Variable taux } \\
\text { de chômage rural }\end{array}$ \\
\hline \multirow{6}{*}{$\begin{array}{l}\text { Variables } \\
\text { exprimant } \\
\text { le taux } \\
\text { de } \\
\text { demande } \\
\text { aux: }\end{array}$} & Aides directes & 0,8 & 0,09 & 0,1 \\
\hline & $\begin{array}{l}\text { Aide à la } \\
\text { modernisation (2007) }\end{array}$ & 0,8 & $-0,6$ & 0,31 \\
\hline & $\begin{array}{l}\text { Aide départ } \\
\text { en préretraite (2007) }\end{array}$ & 0,5 & $-0,4$ & 0,37 \\
\hline & $\begin{array}{l}\text { Aide jeunes } \\
\text { agriculteurs (2008) }\end{array}$ & 0,4 & $-0,3$ & 0,08 \\
\hline & $\begin{array}{l}\text { Aides à la } \\
\text { diversification (2008) }\end{array}$ & 0,6 & $-0,4$ & 0,15 \\
\hline & $\begin{array}{l}\text { Aides agro-environ- } \\
\text { nementales (2007) }\end{array}$ & 0,8 & $-0,7$ & 0,48 \\
\hline
\end{tabular}

Sources: GUS 2010, ARIMR 2008, calcul auteures

(*) Le calcul de la main-d'œuvre est basé sur les statistiques du GUS, qui prennent en compte le nombre de personnes actives cotisant au système de sécurité sociale agricole polonais (le célèbre KRUS, calqué sur le système français de la mutualité sociale agricole). En l'absence ou presque d'indemnités chômage en Pologne, il est plus avantageux lorsque c'est possible de se replier (Maurel et al., 2003) sur l'exploitation en période de chômage que de se déclarer chômeur : on bénéficie ainsi d'une couverture sociale convenable à un tarif avantageux, même si la quantité de travail sur l'exploitation ne nécessite pas d'apport de main-d'œuvre supplémentaire. C'est le " chômage caché » en agriculture qui caractérise certaines régions polonaises à fort taux de chômage rural.

élevée des exploitations et taux élevé de demandes d'aides à la modernisation reflète l'efficacité de la mise en œuvre de cet élément de la politique de compétitivité telle qu'exprimée dans le sens 2 (dominant) $P M$ de notre tableau 2.

Plus surprenant, les aides agro-environnementales semblent demandées, surtout dans les régions de grandes exploitations. Ces aides ne vont pas non plus, par conséquent, aux régions où la main-d'œuvre agricole est la plus élevée par hectare, autrement dit aux régions dont l'agriculture assure la plus forte fonction sociale et est identifiée comme bénéfique pour l'environnement. On trouve ici une illustration du sens $D E n v$ attribué dans les textes aux termes « durables/durabilité » : cette durabilité est soutenue sur le plan environnemental, sans lien avec les aspects sociaux, lien qui aurait été reflété par une forte corrélation avec la variable densité de main-d'œuvre.

De même, sur le plan social, les aides à la diversification des activités rurales, en principe destinées aux plus petites exploitations dans les régions présentant un fort taux de chômage rural, semblent selon nos données mieux captées dans les régions de grandes

\section{Tableau 4. Impact démographique et économique de notre échantillon d'aides}

\begin{tabular}{l|c|c}
\hline & $\begin{array}{l}\text { Taux de demande de l'aide en } \\
\text { 2007 en \% du nombre total } \\
\text { d'exploitations }\end{array}$ & $\begin{array}{l}\text { Enveloppe financière en PLN } \\
\text { versée en } 2006 \text { ou } 2007\end{array}$ \\
\hline Aide unique 1er pilier & $56,89 \%$ & $(2007) 36878533000$ \\
\hline $\begin{array}{l}\text { Ensemble des mesures } \\
\text { développement rural }\end{array}$ & & $(2007) 9657686782$ \\
\hline Aide modernisation & $0,86 \%$ & $(2006) 362400000$ \\
\hline Préretraite & $0,38 \%$ & $(2006) 192700000$ \\
\hline Installation & $0,28 \%$ & $(2006) 144200000$ \\
\hline Diversification des activités & $0,17 \%$ & \\
\hline Mesures agri-environnementales & $0,9 \%$ & $(2006) 2282000000$ \\
\hline
\end{tabular}

Sources: ARIMR 2010, Calculs des auteures, Eurostat 2010

(http://eur-lex.europa.eu/budget/data/D2007_VOL4/PL/nmc-titleN123A5/index.html) 


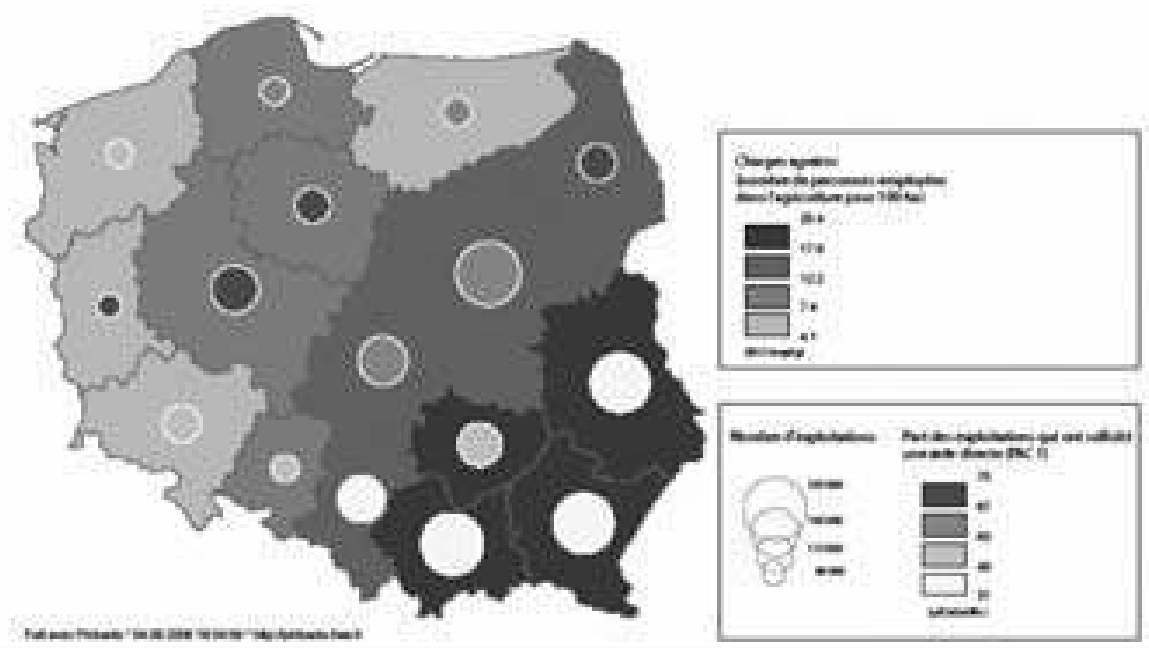

Sources : ARIMR 2010, Calculs des auteures, Eurostat 2010

exploitations, sans lien effectif avec le taux de chômage rural ou la densité régionale de main-d'œuvre agricole. L'aide manque nettement sa cible et paraît captée par des zones du pays qui en ont moins besoin.

Pour les aides jeunes agriculteurs en revanche, que l'on pourrait appréhender comme une aide relevant de la logique modernisatrice $P M$ de notre tableau 2 compte tenu de ses conditions d'application, l'hypothèse d'une corrélation entre taux de demande et taille moyenne des exploitations, quantité de main-d'œuvre agricole ou taux de chômage rural ne tient guère : il faudra chercher ailleurs les moteurs de la constitution de ces dossiers par les jeunes agriculteurs. D'une certaine manière, cette aide également semble manquer sa cible.

Le commentaire des éléments budgétaires impose une forte nuance à cette analyse : alors que l'aide unique du premier pilier a été demandée par environ la moitié des exploitations ${ }^{6}$, les cinq aides du second pilier que nous considérons ici ont concerné chacune moins de $1 \%$ des exploitations du pays en 2007, trois ans après leur mise en place.

6. Toutes exploitations confondues à partir de 1 ha : ce pourcentage reste encore faible.
Le fait que des aides soutenant potentiellement la durabilité des exploitations manquent leur cible et soient en pratique captées par les voïvodes (et sans doute les exploitations) les moins paysannes est un premier constat d'échec : mais leur faible impact démographique au cours de leurs premières années de mise en œuvre limite toute conclusion sur leurs effets, sans commune mesure avec l'impact potentiel du premier pilier de la PAC, mieux capté par l'ensemble des exploitations et doté d'une enveloppe financière environ quatre fois supérieure à celle du second pilier lors de leurs premières années de mise en place.

Les mises en carte (voir carte 1) des données sur ce premier pilier de la PAC polonaise font apparaître de forts contrastes régionaux.

Les cinq voïvodies les plus paysannes ${ }^{7}$ dont l'agriculture est la plus « sociale » ${ }^{8}$

7. Podkarpackie, Malopolskie, Swietokrzyskie, Sląskie, Lubelskie.

8. La quantité de main-d'œuvre y est toujours supérieure à 17 actifs pour 100 ha contre 4 actifs pour 100 ha dans la voïvodie qui représente l'autre extrême de l'échelle; les exploitations disposent de 3 à 6 ha en moyenne contre 12 à 20 ha dans les 5 voïvodies polonaises présentant les tailles moyennes les plus importantes. 


\section{Carte 2. Premières attributions d'aides directes en Roumanie}

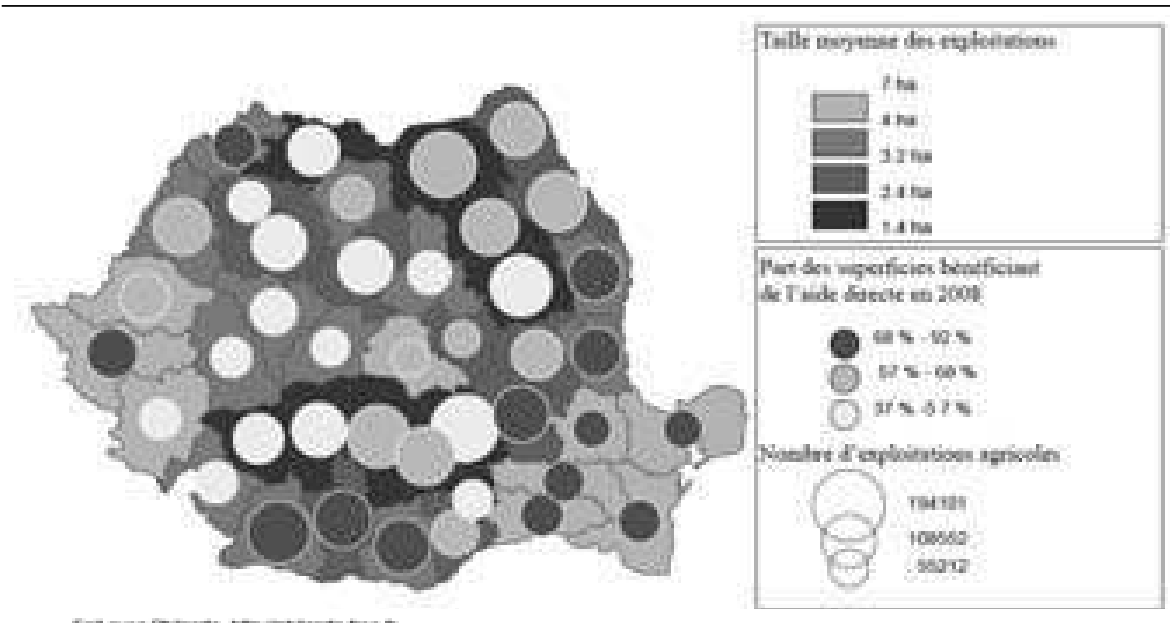

Source : Agentia de Plata si Interventie pentru Agricultura, 2008

sollicitent beaucoup moins qu'ailleurs l'aides du premier pilier de la PAC, ainsi que l'aide à la modernisation des exploitation. Autrement dit, et c'est important même si on pouvait l'anticiper, cette ligne de clivage social est désormais potentiellement accentuée par les aides tendant à favoriser les exploitations qualifiées de compétitives et les régions où ces exploitations sont les plus représentées.

\section{Les effets de ciblage régional des dispositifs d'aide en Roumanie}

En Roumanie, les politiques sont mises en œuvre avec un décalage de près de trois ans et les données ne sont encore que partiellement disponibles. Mais on peut d'ores et déjà anticiper sur des logiques similaires à celles observées en Pologne.

L'analyse des aides du premier pilier donne une première image des disparités. Le premier pilier perçoit 1,7 milliard (près d'un milliard est affecté aux aides directes simplifiées et le reste aux interventions sur les marchés). L'essentiel des aides va toutefois au second pilier qui bénéficie de plus de 2,3 milliards d'euros pour les deux premières années. C'est l'axe 1 destiné à accroître la compétitivité qui est le premier bénéficiaire de cette manne financière avec $44 \%$ des fonds. Le deuxième axe destiné à la valorisation agro-environnementale des espaces défavorisés et des paysages obtient près de $26 \%$ du total des fonds publics alloués. L'axe 3 qui vise la diversification des économies rurales et la modernisation des équipements et services villageois (adduction d'eau et voirie principalement) peut compter sur $28 \%$ des fonds prévus ${ }^{9}$.

La carte de la sollicitation des aides directe dessine une géographie de la demande inscrite dans les grandes aires de plaine céréalières : le Baragan et la Doubroudja au sud-est, le Banat et la plaine du Cris à l'ouest, secondairement la plaine danubienne oltenienne et les périphéries moldaves. Ce sont les espaces où les grandes exploitations sociétaires tiennent l'essentiel des surfaces. À l'inverse, les campagnes de l'arc carpatique (et notamment l'Oltenie des piémonts) mais aussi les bassins de Transylvanie peinent à capter les aides. Les taux faibles observés dans les campagnes paysannes s'expliquent d'abord par l'exclusion de la mesure des micro-

9. Pour le détail des différentes priorités voir Hirschhausen (2008). 
RECHERCHES

Catherine DARROT, Béatrice Von HIRSCHHAUSEN Avec la collaboration de Marie-Luce GHIB

exploitations. Le ciblage des aides sur les exploitations de plus de 1 ha et sur les parcelles de plus de 0,3 ha vise à limiter le saupoudrage d'aides aux montants dérisoires, distribuées à une multitude de micro exploitations. Ce ciblage est aussi conforme à un schéma qui considère implicitement que les plus petites exploitations sont vouées à une disparition prochaine : aucun dispositif d'aide ne leur est donc destiné (Ghib, 2011). Ce faisant il exclut une part importante des familles paysannes : la seule limite de 1 ha pour les exploitations exclut à elle seule 49,5 \% d'entre elles et près de $5 \%$ des terres ${ }^{10}$; la limite de 0,3 ha par parcelle exclut en outre des exploitations plus grandes mais excessivement fragmentées, dans un contexte où la redistribution foncière des années 1990 a émietté à l'extrême les parcellaires. La carte laisse supposer que cette sélectivité touche en outre un grand nombre d'exploitations qui sont suffisamment grandes pour pouvoir prétendre aux aides mais qui ne parviennent pas à répondre aux exigences bureaucratiques du programme. Le montage d'un dossier de demande, l'ouverture d'un

10. Données du recensement général de l'agriculture de 2002, (INS 2003). compte bancaire constituent pour bon nombre de familles paysannes un obstacle réel.

Curieusement, ce même critère de taille a été retenu pour des aides agri-environnementales du second pilier spécifiquement destinées aux zones défavorisées ${ }^{11}$. Le programme a ici explicitement visé un développement durable pour des régions qui ne peuvent compter sur la compétitivité de leurs exploitations. Pour l'essentiel, les fonds affectés au second pilier vont donc pour le moment aux mêmes que ceux du premier. L'observation de la mise en œuvre d'une des toutes premières mesures de ce second pilier, destinée à la modernisation des exploitations ${ }^{12}$ montre que comme lors du programme de préadhésion ce sont les grandes entreprises sociétaires ou associatives qui en sont les bénéficiaires quasi exclusifs : l'exploration systématique des listes des entreprises ayant déposé des dossiers lors des premières cessions d'attribution en 2008

11. Il s'agit des mesures de l'axe 2 du Plan national de développement rural 2007-2013 : 211 de soutien à la zone de montagne, 212 de soutien aux zones défavorisées, 214 aux objectifs agro-environnementaux.

12. Il s'agit ici de la mesure 121 du Plan national de développement rural 2007-2013.

\section{Carte 3. Aides à la modernisation et structures agraires en Roumanie}

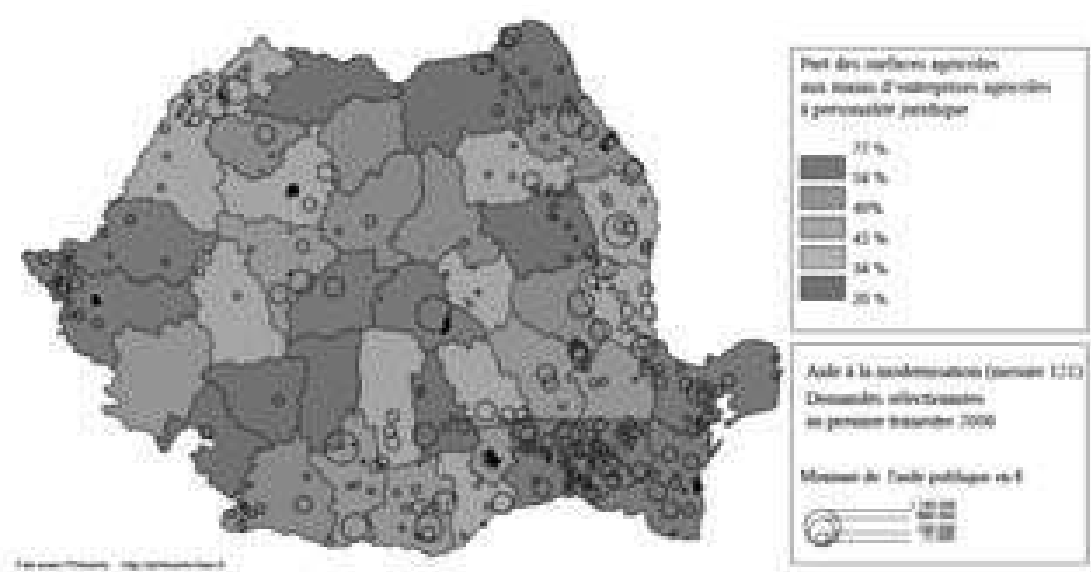

Source : Agentia de Plata pentru Dezvoltare Rurala si Pescuit, 2008 
ne laisse repérer que quelques rares exploitations familiales. Cartographiée, la répartition géographique des projets soutenus reflète celle de l'agriculture d'entreprise (carte 3).

La concentration des grandes entreprises sociétaires dans les grandes plaines du Danube, du Banat ou de Dobroudja permet à ces espaces de solliciter une part importante de l'aide européenne. C'est là que se concentre l'essentiel des aides à la modernisation. Inversement les régions paysannes des collines et piémont du pourtour des Carpates, captent mal la manne européenne : l'émiettement des structures exclut une part importante des terres agricole des aides directes mais également des programmes d'aide à la modernisation et paradoxalement des programmes destinés aux zones défavorisées.

\section{Conclusion}

L'analyse de la mise en œuvre de la PAC dans les deux pays montre que les disparités régionales tendent à se creuser, ainsi que les écarts de développement entre les catégories de structures. Les régions et les exploitations initialement les plus conformes au projet modernisateur et marchand européen, et les plus dotées en termes de capital, captent davantage d'aides européennes, qui viennent alors renforcer le processus de polarisation des structures.

Au vu de ces différents éléments, c'est l'ensemble de la PAC en Pologne et en Roumanie qui semble non seulement valider, mais au demeurant accentuer fortement un processus de polarisation des structures agricoles initialement beaucoup moins spontané que ne le prédirait la perspective de l'économie standard : de 1990 à l'adhésion en 2004, la Pologne s'était par exemple davantage fait remarquer par une stabilité structurelle inédite et inattendue que par un creusement accru et spontané des écarts socio-économiques entre structures et entre régions en faveur des zones appréhendées comme plus compétitives (Darrot, 2008a).
Dans ces conditions, il ne sera pas possible de parler à l'avenir, comme c'est parfois le cas dans certains documents issus de l'économie politique, de sélection naturelle des exploitations les plus compétitives. Si cette sélection a lieu, elle aura relevé d'un choix politique comparable à ce qui fut opéré au cours des Trente Glorieuses au sein des pays fondateurs de l'Union, qui n'est pourtant plus possible aujourd'hui. Pour reproduire un schéma de modernisation et de concentration des structures, analogue à celui des agricultures ouest-européennes des Trente Glorieuses, les politiques ne peuvent plus jouer sur l'attractivité des marchés du travail dans les autres secteurs. Les emplois y sont aujourd'hui trop peu stables pour que micro-exploitations paysannes puissent miser sur une conversion totale et la cession de leurs terres. Les paysans polonais comme plus récemment les paysans roumains ont progressivement développé de puissantes stratégies de résistance aux projets étatiques, dans le but de garantir la subsistance de leur groupe familial quelles que soient les évolutions politiques traversées par leur pays. Ces stratégies sont basées sur l'autonomie économique et technique de la famille, à partir de systèmes productifs et d'activités économiques aussi diversifiés que possible, de solidarités familiales internationales comme locales, et de systèmes d'échanges locaux parallèles aux circuits économiques officiels satisfaisant autant que possible les besoins de services.

En définitive, les objectifs européens de convergence ne se traduisent guère dans la réalité des aides, qui demeurent clairement sélectives en faveur d'une catégorie restreinte d'exploitations. On retrouve à travers les aides observées les principes téléologiques mis en évidence dans l'analyse lexicométrique des textes proposée en première partie.

Ce constat encourage en retour à imaginer la construction d'une politique de développement rural intégrée qui prendrait en 
RECHERCHES

Catherine DARROT, Béatrice Von HIRSCHHAUSEN Avec la collaboration de Marie-Luce GHIB

compte l'ordre des priorités pour les familles rurales, s'accompagnant de politiques macro-structurelles de désenclavement visant à favoriser et renforcer le développement des activités existantes, formelles ou informelles. Ces politiques de développement devraient également reconnaître la présence et l'efficacité des activités de diversification développées dans la sphère informelle de l'entre-soi local (Darrot, op. cit.). Soutenir le foisonnement de ces activités rurales locales pour le moment semicachées, car situées partiellement dans la sphère de l'économie informelle, se révèlerait probablement juridiquement plus délicat, mais socialement et économiquement plus efficace que l'invention ex nihilo de nouvelles pistes de développement économique pour lutter contre le chômage rural et les faibles revenus des propriétaires de petites et moyennes exploitations.

Le modèle ouest-européen, basé dans le domaine agricole sur un modèle familial lieu de promotion de la libre entreprise et sur une agriculture très intégrée au marché, perdrait d'ailleurs paradoxalement moins grâce à de tels choix qu'à travers la politique offensive de réforme des structures et des activités économiques agricoles pour le moment adoptée : cette dernière enclenche en effet le renforcement de processus de repli semi-autarcique (Maurel et al., op. cit.) éprouvés historiquement à l'Est lorsque le contexte politique et économique contrecarre les dynamiques paysannes. 


\section{RÉFÉRENCES BIBLIOGRAPHIQUES}

CE (Commission européenne), (1998). Agriculture situation and prospects in the Central and Eastern European Countries. Direction générale de l'agriculture (DG VI), working document.

CE (Commission européenne), (2002). Agricultural situation in the candidate countries, Country report on Poland. Direction générale de l'agriculture.

Darrot C. (2008a). La voie paysanne polonaise, une chimère structurelle qui déconcerte l'Europe « Centre ». In Gana A., Streith M. (Dir.), «Libéralisation, processus d'intégration régionale et restructurations agricoles au Sud et à L'Est, regards croisés », Revue AutrePart, $\mathrm{n}^{\circ} 46$.

Darrot C. (2008b). Les paysans polonais à l'épreuve de la Politique Agricole Commune Européenne, une analyse multi-disciplinaire d'un référentiel professionnel pour un dialogue de normes. Thèse de doctorat, Agrocampus Ouest, site de Rennes, septembre

Ghib M.-L. (2007). Restructuration foncière de l'agriculture en Roumanie : analyse de la mesure de rente viagère. Mémoire de recherche, Master EGT, Université de Bourgogne, Tapuscrit, $76 \mathrm{p}$.

Ghib M.-L., (2011). Transformation des structures agricoles de production en Roumanie : quelles politiques publiques d'accompagnement? Thèse de doctorat, Agrosup Dijon, 11 juillet

Hirschhausen B. (Von) (1997). Les nouvelles campagnes roumaines : paradoxes d'un retour paysan. Paris, Belin, coll. Mappemonde, $240 \mathrm{p}$.

Hirschhausen B. (Von) (2008). Les sociétés rurales roumaines face à l'irruption de programmes de développement. Revue d'Études comparatives Est-Ouest, vol. 39, $\mathrm{n}^{\circ} 4$, p. 113-142.
INS (Institutul Național de Statistică) (2003). Bucarest, Recensaminte General Agricol 2002.

Maurel M.-C., Hamlaska M., Lamarche H. (2003). Le repli paysan; trajectoires de l'après-communisme en Pologne. Paris, L'Harmattan, coll. pays de l'Est, $252 \mathrm{p}$.

MADR (Ministère de l'Agriculture et du Développement Rural) (2004). Restructuring and modernisation of the food sector and rural development 2004-2006. Varsovie, $166 \mathrm{p}$.

MADR (Ministère de l'Agriculture et du Développement rural) (2005). Rural Development plan for Poland 2004-2006. Varsovie, $206 \mathrm{p}$.

MADR (Ministère de l'Agriculture et du Développement rural) (2007). Rural Development plan for Poland 2007-2013. Varsovie, 206 p.

MADR (Ministère de l'Agriculture et du Développement Rural) (2008). Programul Național de Dezvoltare Rurală 20072013. versiune consolidată 08 februarie, Număr CCI 2007RO06RPO001, 815 p.

MAFRD (Ministry of Agriculture, Forests and Rural Development) (2006). National Plan for Agriculture and Rural development over the 2000-2006 period under the EU special accession program for agriculture and rural development (SAPARD). Revised version, 7th of July, $650 \mathrm{p}$.

Rey V., Groza O., Ianos I., Patroescu M. (dir.) (2007). Atlas de la Roumanie. Paris, La documentation française/CNRSGDRE S4, coll. Dynamique du territoire, 211 p., 250 cartes, 24 graphiques et 1 tableau ( $2^{\mathrm{e}}$ édition). 